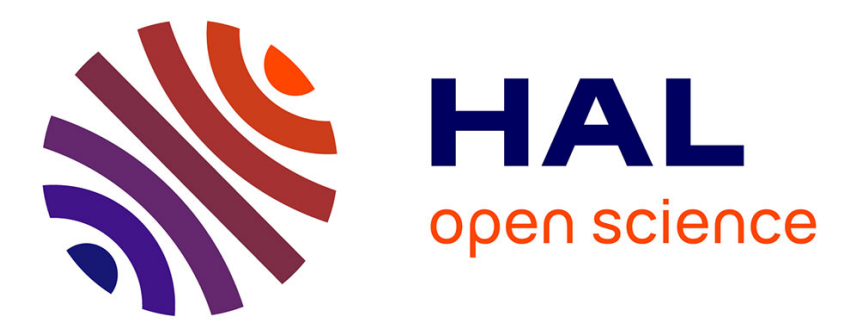

\title{
Local damage detection by nonlinear coda wave interferometry combined with time reversal
}

Nikolay Smagin, Andrey Trifonov, Olivier Bou Matar, Vladislav Aleshin

\section{To cite this version:}

Nikolay Smagin, Andrey Trifonov, Olivier Bou Matar, Vladislav Aleshin. Local damage detection by nonlinear coda wave interferometry combined with time reversal. Ultrasonics, 2020, 108, pp.106226. 10.1016/j.ultras.2020.106226 . hal-03002280

\section{HAL Id: hal-03002280 \\ https://hal.science/hal-03002280}

Submitted on 24 Jun 2021

HAL is a multi-disciplinary open access archive for the deposit and dissemination of scientific research documents, whether they are published or not. The documents may come from teaching and research institutions in France or abroad, or from public or private research centers.
L'archive ouverte pluridisciplinaire HAL, est destinée au dépôt et à la diffusion de documents scientifiques de niveau recherche, publiés ou non, émanant des établissements d'enseignement et de recherche français ou étrangers, des laboratoires publics ou privés. 


\section{Paper Highlights}

- A new non-destructive testing method for localized damage detection in complex structures is proposed. It is combined of non-linear coda wave mixing and time reversal techniques.

- Time-reversal pump is possible only in pulsed mode due to spatio-temporal wave compression. Features of coda wave mixing in continuous and pulsed pump mode are considered. It has been experimentally observed that an aftereffect of a pulsed pump provides a nonlinear interaction between pump and probe waves of an overall level sufficient for a defect detection.

- Non-monotonous behavior of time-windowed delay and correlation coefficient in an entirely thermally-shocked glass sample is reported. Contrary to the precedent case, the behavior of these parameters in a locally damaged glass sample is monotonous.

- Localization of a defect in a glass sample using a one-channel time reversal system and nonlinear coda wave interferometry has been demonstrated. 


\title{
Local Damage Detection by Nonlinear Coda Wave Interferometry Combined with Time Reversal
}

\author{
N. Smagin ${ }^{1}$, A. Trifonov ${ }^{1}$, O. Bou Matar ${ }^{1}$, V.V. Aleshin ${ }^{1,2, *}$ \\ ${ }^{1}$ Univ. Lille, CNRS, Centrale Lille, ISEN, Univ. Valenciennes, UMR 8520 - IEMN, LIA \\ LICS/LEMAC, F-59000 Lille, France \\ ${ }^{2}$ Tomsk State University, 634050, Tomsk, Russia \\ *corresponding author: vladislav.aleshin@univ-lille.fr
}

\begin{abstract}
Coda wave interferometry (CWI) is a sensitive ultrasound method for detection of weak and local changes in complex inhomogeneous media. In a nonlinear modification of the method discussed here, a high-frequency probe coda is compared to its replica obtained in the presence of lowfrequency pumping. If after the filtering out of low frequencies the coda signals are different, this is attributed to nonlinear pump-probe interaction induced by contact acoustical nonlinearity in the damaged zone. Actually, the CWI methods are used for global inspection of complex media, such as, for example, concrete structures. In this work, a step forward is made; it consists in combining the CWI with the time reversal (TR) technique in order to allow one to focus the pump wave on a selected area in the structure and to detect and localize a flaw. Time-reverse pump is possible only in pulsed mode due to spatio-temporal wave compression. By this reason, the particularities of coda wave mixing in conventionally used continuous and pulsed pump mode are considered. It has been experimentally observed that an aftereffect of a pulsed pump provides a nonlinear interaction between pump and probe waves of a sufficient overall level for a defect detection with TR. Finally, it was shown that a TR focusing even with the minimal available quality i.e. with only one transducer produce a sufficient contrast allowing to distinguish intact and damaged zones with nonlinear CWI.
\end{abstract}

Keywords: coda wave interferometry, nondestructive testing, time reversal, pump-probe experiment

\section{Introduction}

In many cases, ultrasound propagation in weakly attenuating materials results in appearance of so-called coda waves. They are created by interference of acoustical waves repeatedly sampling the same region in space due to multiple scattering produced by inhomogeneities in the material or 
multiple reflections from sample's borders. A coda wave recorded by an acoustical receiver represents a quasi-chaotic signal having, however, a deterministic nature [1]. The coda signal is extremely sensitive to any subtle changes in material's geometry or properties. In other words, during multiple reverberations or scattering, the information about these changes is accumulated and finally becomes measurable [2]. In some sense, the material works as a natural interferometer; correspondingly, the method that uses the high sensitivity of coda waves to changes in material's properties is called the Coda Wave Interferometry (CWI).

For this reason, CWI has found numerous applications is non-destructive testing (NDT) of complex media where conventional techniques in majority of cases are ineffective. The numerous implementations of CWI can be found in the domain of concrete structures monitoring [3-5] exhibiting substantial scattering and attenuation or in complex industrial conditions [6]. It was shown that CWI is able to measure relative velocity change with a high precision of $10^{-5}$ [7].

CWI belongs to the class of baseline NDT techniques consisting in comparison of the initial and potentially modified states of a structure under-test. This point may represent a considerable limitation of the CWI for NDT applications. Coda waves are sensitive to uncontrollable ambient conditions (temperature or humidity changes) making it difficult to distinguish whether the modification in coda wave signal is due to flaw formation in the structure or the to the change of external context $[8,9]$.

By combining the advantages of CWI with nonlinear ultrasonic techniques, a new method called nonlinear coda wave interferometry has been recently proposed in [10]. In this case, a low frequency pump wave is excited in the sample while the corresponding low-frequency component in the coda signal is filtered out. If coda signals produced with and without pump wave are still different, the deviation can be only attributed to nonlinear frequency mixing due to contact acoustical nonlinearity that, in turn, reveals the presence of damage.

The nonlinear CWI technique is substantially more selective to damage than the linear counterpart since damage affects the nonlinear properties in a much greater degree than the linear ones. In addition, the nonlinear version of the method does not require any baseline coda data that can be influenced by environmental changing or aging.

By default, the linear or nonlinear CWI alone does not provide any opportunity of localizing defects. One of the ways to assure this opportunity in linear CWI is to combine measurements with different pairs of sensors and numerical modelling [11,12]. Another way to assure a localization ability in nonlinear CWI is to focus energy in a known point inside the test sample. The nonlinear modulation process requires the presence of both the probe and the pump acoustic strain at a defect location in order to work properly. It is supposed that nonlinear interaction will be stronger when pump is focused on the defect thus providing a localization possibility. 
Focusing could be effectuated with phased arrays [13] as well as by spatial mechanical scanning [14]. Another opportunity is offered by the Time Reversal (TR) method in acoustics that is based on the fact that the waves created by emission of time-reversed signals focuses back on the receiver [15]. The acoustical energy compression is made both in time and space, regardless of the position of the initial source and of the heterogeneity of the medium in which the wave propagates. In comparison to traditional beamforming techniques using, in particular, phased arrays, TR is more versatile since, among other things, to focus a specific wave field component [14]). TR also requires less prior information about the test sample, but demands additional steps for operation. Several non-linear TR-based techniques for NDT has been proposed [16,17]. In [18] a non-linear defect localization was performed with a 6-channel TR nonlinear by elastic wave spectroscopy process. Additionally, flaw localization with non-linear modulation has been reported basing on resonance patterns [19].

In this work, we propose a method for local structure inspection using nonlinear mixing of a probe coda wave with a TR-focused pump wave. Both CWI and TR techniques are well adapted for application in complex heterogeneous media where conventional linear NDT methods can fail or be too difficult to implement. Typical examples of such heterogeneous media are the modern composite materials used in airplane constructions. Our objective here is to explore the potential of a new technique combining these two methods for structural health monitoring.

Features of coda wave mixing in continuous (used conventionally) and pulsed pump mode were considered. It has been experimentally observed that an aftereffect of a pulsed pump provides a nonlinear interaction between pump and probe waves of an overall level sufficient for a defect detection. The differences of pulsed and continuous mode has been already considered in [20] but for the case of linear CWI with amplitude and phase lock-in measurements for a global inspection. Finally, a localization of a defect in a glass sample using a one-channel TR system and non-linear CWI has been demonstrated.

\section{Nonlinear coda wave interferometry}

\subsection{Principle}

In many cases, sound waves propagating in a solid material are many times scattered by material's inhomogeneities and reflected from sample's boundaries. In reverberating or multiscattering media with relatively low attenuation, a measured acoustic echo typically consists of three parts: ballistic (or direct) waves, coda waves, and noise. Ballistic waves are first to arrive as they propagate directly from the source to the sensor. Coda waves are a later part of the signal that 
corresponds to a diffused field having an exponentially decaying amplitude. Finally, noise is a concluding part of the reverberation appearing when the excitation signal is completely attenuated.

Coda wave interferometry is especially sensible to the presence of damage when used in a nonlinear modification [21] based on interactions between a high-frequency (HF) reverberation signal and a powerful low-frequency (LF) pumping. Firstly, HF reverberations are recorded in the absence of pumping. Secondly, the same HF signal is excited simultaneously with the pump wave. Then low frequencies are filtered out of the coda signal. If the experiment is performed correctly, any difference between the two codas can be attributed only to nonlinear interactions between HF and LF waves i.e., in a linear material, to the presence of damage. In the process of coda formation, tiny changes are accumulated and become observable.

Typical reverberation signals are shown in Fig. 1(a). Two time windows illustrate the ballistic propagation part (Fig. 1(b)) in which small changes between two signals are barely visible, and the coda waves in which they are clearly distinguishable (Fig. 1(c)). The signals have been obtained on a locally damaged glass sample in absence and presence of a LF pumping; the test samples and experimental configuration are described in Section 3.

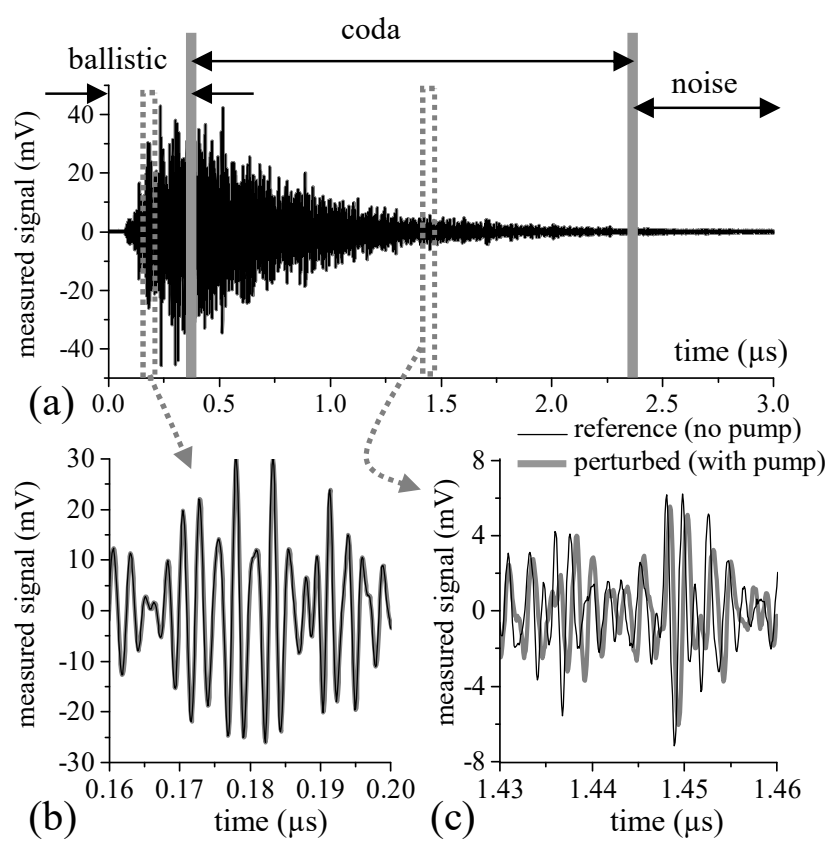

Fig. 1. Experimental reverberation signals recorded on a locally damaged glass sample. The reference signal is recorded in the absence of pumping; the perturbed one is obtained by the nonlinear interaction with a LF acoustic pump.(a). Full reverberation signal containing the ballistic, coda, and noise parts. In the ballistic phase (b), the reference and perturbed signals practically coincide whereas in the coda part (c) the difference becomes important. 
Despite their chaotic appearance, coda waves are highly repeatable so that if no change occurs in the medium over time, the waveforms remain almost identical. The CWI methods assume the multiple scattering medium to act as an interferometer. Long and complex propagation paths of coda waves make it possible to amplify the influence of small propagation medium perturbations often undetectable with conventional pitch-catch techniques.

\subsection{Investigative and localization regimes}

We conducted our nonlinear CWI experiments in two different regimes. In the investigative regime, only the presence or absence of damage is detected while its geometric localization is not considered [10].

In this work, we try to make a step forward by proposing a local inspection option. For revealing a particular position of damage, it is necessary to focus the pump wave at a given position in the sample. Then the measured nonlinear response can only be due to damage occurring at that location whereas the presence or absence of damage outside of the focus pays a negligible contribution. By changing the focus position, it is possible to interrogate the entire sample and see where exactly the damage is located.

Focusing of the pump wave acoustic energy is possible via a procedure called Time Reversal based on reciprocity and time reversal invariance of the wave equation in lossless heterogeneous stationary media. The time reversal invariance means that if a signal $s_{\text {pump }}(t)$ emitted at point $\mathrm{E}$ engenders a response $r_{\text {pump }}(t)$ at point $\mathrm{R}$, then re-broadcasting $q_{\text {pump }}(t)=r_{\text {pump }}(-t)$ at point $\mathrm{R}$ generates the same signal $s_{\text {pump }}(t)$ at the point $\mathrm{E}$ where it was initially excited. Moreover, due to the reciprocity principle, the signal $q_{\text {pump }}(t)=r_{\text {pump }}(-t)$ can be excited at the initial excitation point $\mathrm{E}$ to produce $s_{\text {pump }}(t)$ at the receiver R. Such a configuration is more suitable for nondestructive testing since both broadcasted and re-broadcasted signals are generated by the same transducer. To focus acoustic energy, a short pulse $s_{\text {pump }}(t) \approx \delta(t)$ should be used.

In our experiments we use the inverse filtering technique that represents a modification of the TR proposed in [22]. To get even better focusing at the reception point, it has been suggested to re-broadcast

$$
q_{\text {pump }}(t)=F T^{-1}\left[R_{\text {pump }}^{-1}(\omega) S_{\text {pump }}(\omega)\right]
$$


where $S_{\text {pump }}(\omega)=F T\left[s_{\text {pump }}(t)\right], R_{\text {pump }}(\omega)=F T\left[r_{\text {pump }}(t)\right]$, with $F T[\cdot]$ and $F T^{-1}[\cdot]$, the direct and inverse Fourier transforms, respectively. The implementation details such as dealing with $R_{\text {pump }}^{-1}(\omega)$ in frequency ranges where $R_{\text {pump }}(\omega)$ is close to 0 can be found in [23].

\section{Experimental setup and samples}

\subsection{Probe and pump circuits}

The experimental setup contains two circuits, the probe and the pump (drawn respectively in thin black and in thick grey lines in Fig. 2). The probe signal $s_{\text {probe }}(t)$ represents a sequence of chirps of $200 \mu$ s duration with frequencies between $0.5-1 \mathrm{MHz}$. It is synthesized on a PC and is then sent to the memory of the arbitrary waveform generator Tektronix AWG710B (AWG \#1 in Fig. 2). Further, the probe is amplified with Amplifier Research type 100A400 (Power Amplifier \#1) unit $(100 \mathrm{~W})$ and is emitted in the test sample by an ultrasonic transducer. The measured reverberation response is recorded by the LeCroy $64 \mathrm{Xi}$ oscilloscope connected to the probe receiver on the opposite corner of the plate. In the received signal, frequencies below $0.5 \mathrm{MHz}$ are filtered out by a $7^{\text {th }}$ order Chebyshev analog high-pass filter (HPF) in the purpose of excluding low frequency pump components.

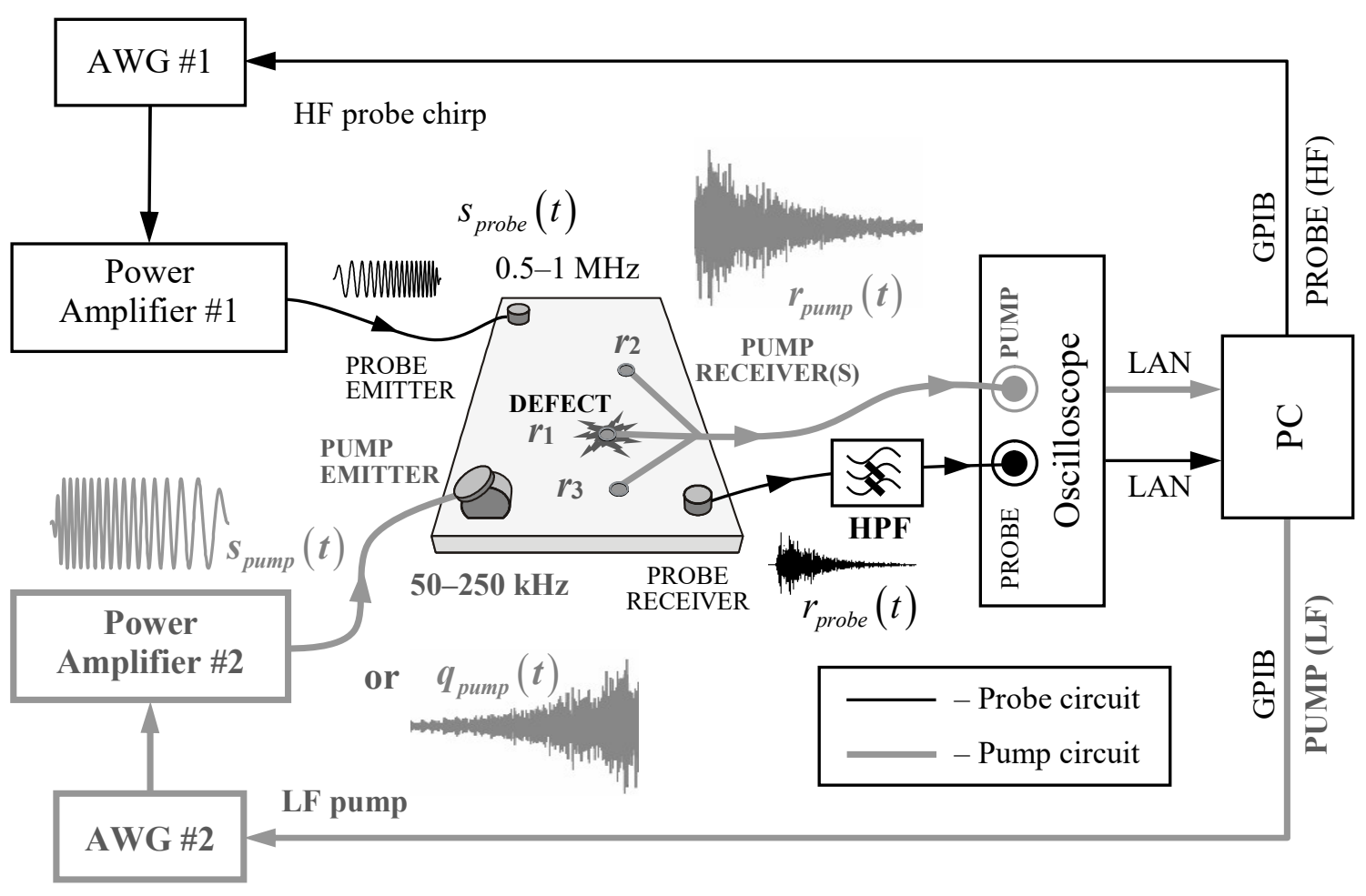

Fig. 2. Scheme of the experimental setup with pump (thick gray) and probe (thin black) circuits. 
The pump signal is excited by the Tektronix AWG710 (marked AWG \#2 in Fig. 2) generator connected to the Amplifier Research 150A250 (Power Amplifier \#2) $150 \mathrm{~W}$ device which allows obtaining $150 \mathrm{~V}$ of peak amplitude on a capacitive load. The amplifier's gain could be controlled remotely.

In the investigative regime, only the HF coda $r_{\text {probe }}(t)$ is measured and analyzed, whereas in the localization regime LF measurements in the sample are also required. The latter is performed by three pump receivers $\left(r_{1}-r_{3}\right)$; one of them $\left(r_{1}\right)$ is positioned directly on the defect while the other two are located away from it (see Fig. 2). Each pump receiver measures an LF reverberation signal $r_{\text {pump }}(t)$ that represents a reaction of the propagation medium on the excitation $s_{\text {pump }}(t)$ produced by the pump emitter. The coda LF $r_{\text {pump }}(t)$ is required for the calculation of $q_{\text {pump }}(t)$ via Eq. (1). Then $q_{\text {pump }}(t)$ is excited by the same pump emitter to produce so-called retro-focusing of acoustic energy at the pump receiver on which $r_{\text {pump }}(t)$ was measured. The second pump wave emission is accompanied by the generation of a HF signal $s_{\text {probe }}(t)$ by the probe emitter and recording of the HF coda $r_{\text {probe }}(t)$ by the probe receiver. The above measurements are repeated in the presence and in the absence of the pump and for each pump receiver $r_{1}-r_{3}$. The difference between the reference and the perturbed codas indicates the presence of damage at the location of the pump receiver.

\subsection{Test samples}

In our experiments, we use two samples: an entirely damaged one with highly nonlinear properties allowing easer detection, and a sample with localized impact damage (including its intact counterpart).

The entirely damaged sample represents a non-rectangular glass plate (approximately $20 \times 15 \times 1 \mathrm{~cm}^{3}$ ) everywhere damaged by multiple cracking appeared as a result of a thermal shock (Fig. 3 (a)). The cracks are approximately uniformly distributed over the sample's volume and allow one obtaining strongly nonlinear CWI signals in the investigative regime. 


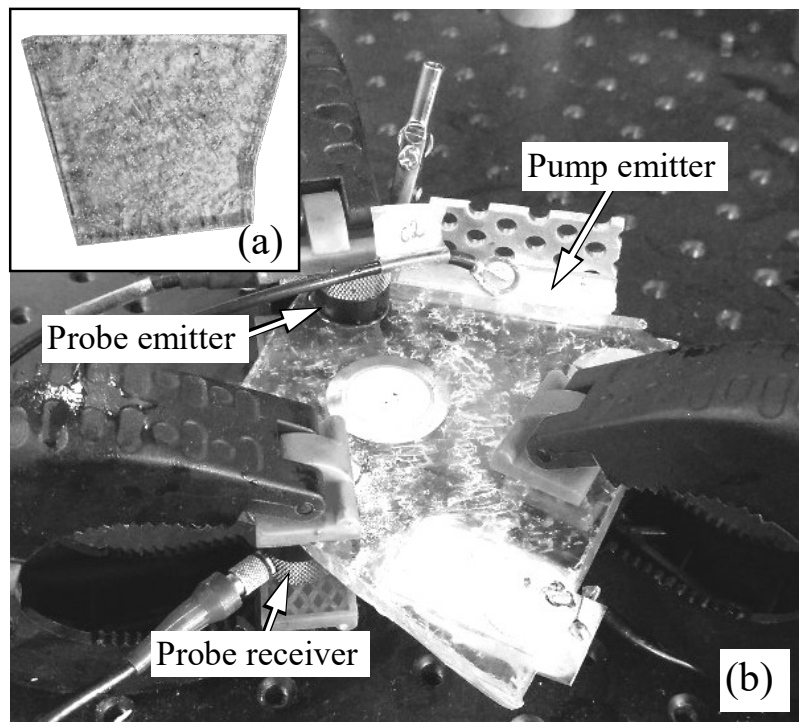

Fig. 3. Entirely damaged sample (a) and configuration (b) of emitters and receivers.

For the pump injection, a rectangular PZT plate of dimensions $45 \times 15 \times 1.5 \mathrm{~mm}^{3}$ was coupled to sample from lateral side (Fig. 3(b)) using a cyanoacrylate adhesive. Probe emission and reception was provided with two identical Panametrics V103-RM (central frequency $1 \mathrm{MHz}$ ) contact transducers, clamped at the opposite sides of the plate. The exact positions of transducers do not have a significant influence on the results (see also [10]).

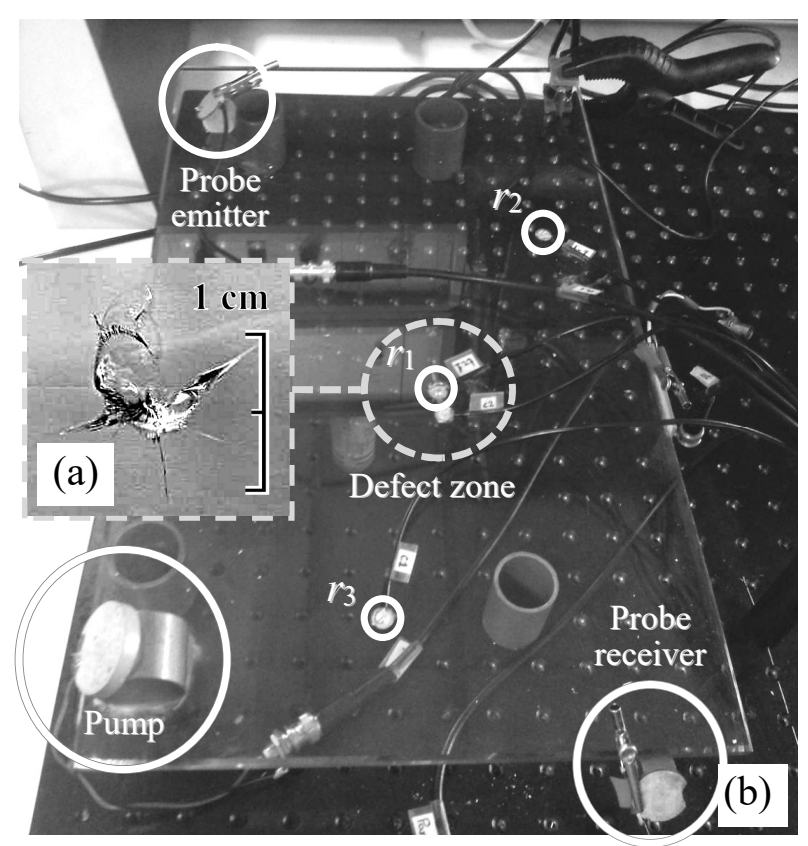

Fig. 4. Zoomed impact damage (a) in the locally damaged sample and configuration (b) of emitters and receivers.

A locally damaged sample represents a glass plate $\left(30 \times 50 \times 1 \mathrm{~cm}^{3}\right.$, Fig. $\left.4(\mathrm{~b})\right)$ in which a flaw of about of $1 \times 1 \times 0.5 \mathrm{~cm}^{3}$ was produced by a point mechanical impact (Fig. 4(a)). This sample was 
used in order to perform focused pump CWI experiments and estimate the localization capabilities of the method.

The LF pump waves were emitted by a PZT-27 disk of $40 \mathrm{~mm}$ diameter and $17.5 \mathrm{~mm}$ thickness $(87 \mathrm{kHz}$ fundamental resonance frequency). At the same time, in the purpose of demonstrating the localization capability, the acquisition of the LF pump codas should be performed at various locations in the sample and in a point manner e.g. by a laser vibrometer [24] or ultrasonic pinducer (needle transducer). In the described case, $10.5 \mathrm{~mm}$ diameter $(8 \mathrm{kHz}$ central frequency) radially polarized KEPO FT-10.5 piezo-disks have been glued to the glass plate at the location of the defect and in several arbitrary locations marked $r_{1}, r_{2}$ and $r_{3}$ in Fig. 4 (b) and in Fig. 2. As the reception is carried out in a frequency range far away from the resonance frequency, their transfer function is nearly constant in the used spectrum region. By means of the re-broadcasting procedure with the LF signals recorded at $r_{1}, r_{2}$ and $r_{3}$, we managed to consecutively create focal spots of about of $1 \mathrm{~cm}$ around these locations which has been verified by additional measurements with a Valpey-Fisher Pinducer VP-0.5. The value of $1 \mathrm{~cm}$ approximately corresponds to the acoustic wavelength in glass under selected frequencies as well as to the defect size. In such a configuration, obtaining of a pronounced nonlinear signal at the defect position $r_{1}$ and the absence of such a signal at other spots $r_{2}$ and $r_{3}$ would be a proof of the damage localization capability.

As for the entirely damaged sample, two Panametrics V103-RM contact transducers have been clamped on the plate in the purpose of the probe signal emission/reception.

In samples with regular geometric shapes, the time reversal inverse focusing procedure may encounter difficulties due to formation of regular resonance patterns [25]. In other words, Eq. (1) guarantees sharp focusing only in highly reverberating geometries [26] in which a diffused acoustic field is easily formed. To introduce more reverberations, the pump transducer was coupled to a metallic chaotic cavity [27] using a cyanoacrylate adhesive. The cavity had a form of truncated $40 \mathrm{~mm}$ diameter cylinder (see "Pump" in Fig. 4 (b)).

An intact glass plate of the same dimensions $\left(30 \times 50 \times 1 \mathrm{~cm}^{3}\right)$ was used as a reference. In the absence of non-linear scatterers, a system is expected to exhibit a linear behavior with no nonlinear coda wave mixing.

With these samples, three experiments have been performed. The first one on the entirely damaged sample with high nonlinearity was conducted as a test for the investigative regime in continuous and pulsed pump modes. The objective of second one was to reproduce the results of [10] by detecting the presence of damage in a locally damaged sample by means of the investigative regime. Finally, the third experiment has shown a potential of the new localization technique based on the nonlinear CWI in combination with retro-focusing via time reversal. 


\section{Investigative CWI regime on the entirely damaged sample}

\subsection{Pump and probe signals in the pulsed mode}

The pump wave should excite the entire sample in a more or less uniform manner, without having pronounced nodes of resonance patterns. In a highly scattering media, it is enough to use a sinewave excitation; for this reason, a series of tone bursts was used (at $76.88 \mathrm{kHz}$ of duration $\tau_{\text {pump }}=800 \mu \mathrm{s}$ spaced in time by $T_{\text {pause }}=99.2 \mathrm{~ms}$, see $s_{\text {pump }}(t)$ in Fig. 5 (a)). In order to have a rich reverberation pattern at many high frequencies, the probe signal used was a sequence of $0.5-1 \mathrm{MHz}$ chirps of duration $\tau_{\text {probe }}=200 \mu \mathrm{s}$ repeated at the same period $T_{\text {probe }}=T_{\text {pump }}=100 \mathrm{~ms}\left(\right.$ see $s_{\text {probe }}(t)$ in Fig. 5).

In the reported experiments with the use of available equipment, the generation of the LF pump wave was accompanied by a parasite HF component. When the pump transducer is driven at a resonance frequency, multiple higher piezoelement resonance frequencies are also excited. Their frequencies can reach the frequency of the probe wave, pass through the input high-pass filter and mix up with the probe signal. These distortions can be confused with the distortions due to damageinduced nonlinear wave mixing. This difficulty has been circumvented with a compensation technique consisting in measurement of the parasite HF pump generation in the absence of probe, and in subtraction of the measured component from a real signal with the probe being switched on. This technique helps partially to get rid of the parasite component. A more radical solution used was to combine the compensation with another method where the pump and the probe waves are spaced in time (Fig. 5) and interact through an aftereffect induced in the damaged material by pumping. It will be shown below that in the considered pulsed mode the aftereffect arising after each pulse due to a final decay rate of the pump wave is sufficiently strong to be used for damage detection via CWI criterions based on a correlation analysis of the measured data. 


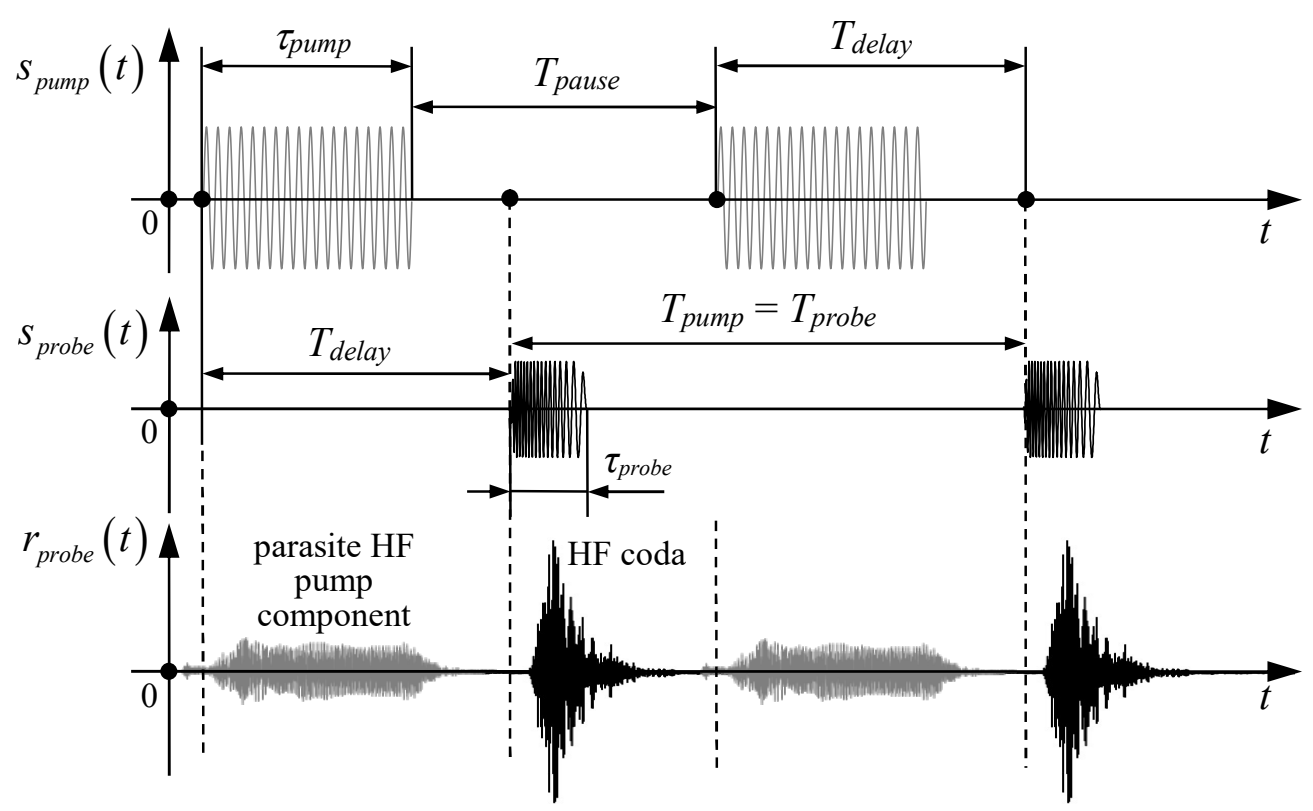

Fig. 5. Illustration for emitted pump and probe signals $s_{\text {pump }}(t)$ and $s_{\text {probe }}(t)$, and for the received coda probe wave $r_{\text {probe }}(t)$ (in black in the presence of the parasite HF pump component (in grey), in the experiment on the entirely damaged sample in the investigative regime (pulsed mode).

\subsection{Correlation analysis}

A typical reverberation signal recorded in this case is shown in Fig. 6(a). For the correlation analysis we select a decaying fragment $45 \mu \mathrm{s}<t<145 \mu \mathrm{s}$ assuming that the increasing part $0<t<45 \mu \mathrm{s}$ corresponds to the less informative ballistic part, while amplitudes less than $50 \mathrm{~dB}$ [28] of maximum are considered as noise.

Two coda waves recorded in two close situations are generally similar, but local fragments can differ i.e. arrive sooner or later or experience time scale distortions (see Fig. 6 (b)). To reflect the local character of these distortions, a windowed correlation coefficient is preferable [2] in which the perturbed coda is compared to a time-shifted initial coda:

$$
C_{T}\left(t_{c}, \tau\right)=\frac{\int_{t_{c}-T}^{t_{c}+T} r_{i}(t+\tau) r_{p}(t) d t}{\sqrt{\int_{t_{c}-T}^{t_{c}+T} r_{i}^{2}(t+\tau) d t \cdot \int_{t_{c}-T}^{t_{c}+T} r_{p}^{2}(t) d t}} .
$$

Here $r_{p}(t)$ is the perturbed measured response, $r_{i}(t)$ is the initial or reference wave field, $\tau$ is the time lag between them, and $t_{c}$ is the center of a time-shifted window of length $2 T$. Using Eq. (2) it is possible to introduce two criterions describing coda wave evaluation [29]. These values are the 
decorrelation coefficient $D_{T}\left(t_{c}\right)$ defined as one minus peak coherence as a function of the window center time $t_{c}$, and $\tau_{T}\left(t_{c}\right)$, an optimal delay as a function of $t_{c}$,

$$
\begin{aligned}
& D_{T}\left(t_{c}\right)=1-\max _{\tau}\left(C_{T}\left(t_{c}, \tau\right)\right), \\
& \tau_{T}\left(t_{c}\right)=\underset{\tau}{\operatorname{argmax}}\left(C_{T}\left(t_{c}, \tau\right)\right),
\end{aligned}
$$

where all maxima are obtained with respect to $\tau$. The decorrelation coefficient has a sense of a measure of the signal shape change, whereas the optimal delay is a measure of the local time shift.

The choice of the time window width $2 T$ is done as a compromise between the following considerations [11]. On one hand, averaging in Eq. (1) means that $2 T$ should be much larger than a period of the highest frequency oscillation in the coda. On the other hand, this averaging is local (windowed) and depend on an additional coordinate $t_{c}$, hence the window width should be much less that the coda duration. In our case, $T=5 \mu$ s was selected.

The criterions $D_{T}\left(t_{c}\right)$ and $\tau_{T}\left(t_{c}\right)$ calculated for the coda waves with/without pumping measured on the entirely damaged sample in the investigative regime are plotted in Fig. 6(c) and (d) as a function the window center $t_{c}$. First of all, it is immediately observed that the presence of the pump wave significantly alters the coda. Indeed, the crack network in the entirely damaged sample (Fig. 3 (a)) is so dense that the glass plate resembles a granular material with a high number of loose contacts. Powerful acoustic loading can completely redistribute the pattern of open and closed cracks in the continuous way i.e. as long as it is applied, similarly to force chain network in granular materials that rapidly alters following an external action [30]. The HF probe propagation with a typical wavelength of about $1 \mathrm{~mm}$ will be strongly affected by the pump is this case, since all paths determined by transitions and reflections from open and closed crack sections are continuously modified. At the moments when the crack network becomes significantly different, $D_{T}\left(t_{c}\right)$ quickly drops (Fig. 6 (c)). However, at some other moments the crack network can be rebuilt and return most likely to the original state, as $D_{T}\left(t_{c}\right)$ can also quickly grow till values of about of $90 \%$. Crack "breathing" also affects the optimal delay $\tau_{T}\left(t_{c}\right)$ which manifests intensive oscillations (Fig. 6 (d)).

We have additionally verified that local heating of the entirely damaged sample provoked the same kind of non-monotonous optimal delay $\tau_{T}\left(t_{c}\right)$. Moreover, the behavior was each time different depending on the localization of heat source. This can be seen as another manifestation of the fact that the dense crack network considerably "weakens" the material so that any perturbation can completely change the configuration of open and closed crack fragments. 

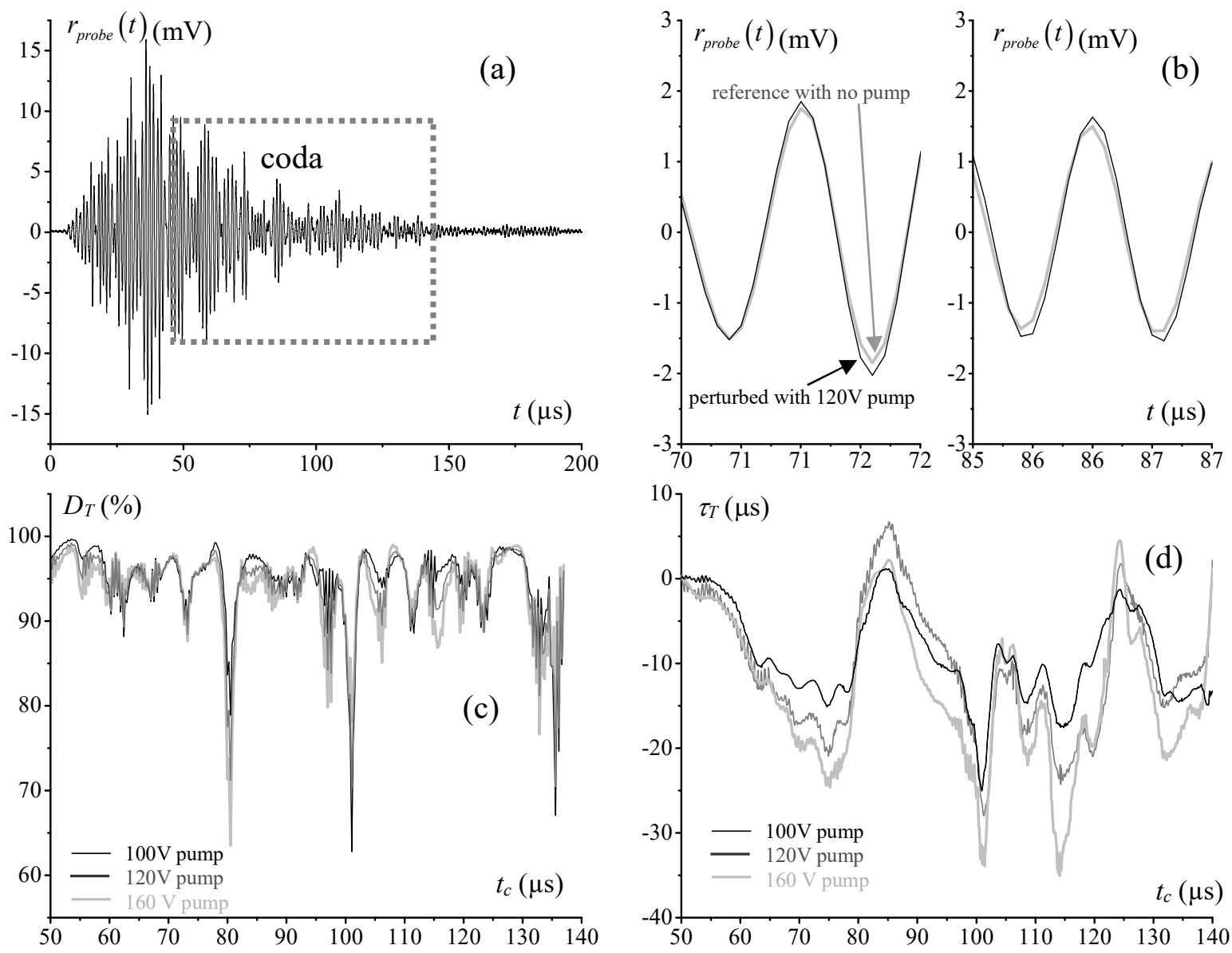

Fig. 6. (a) Full probe reverberation signal obtained on the entirely damaged glass plate (pulsed mode) with its coda part marked with the dashed frame. (b) Two fragments of the coda waves in which the perturbed coda arrives sooner or later than the reference one. (c) Deviations between two coda signal with and without pumping quantified by the curve $D_{T}\left(t_{c}\right)$ calculated using Eq. (3) with a time window of width $T=5 \mu$ s centered at $t_{c}$. (d) The optimal delay that corresponds to maximum correlation; the first quasi-monotonous front in $\tau_{T}\left(t_{c}\right)$ has the inclination $\alpha_{0}$ (not shown) that can be used as a single parameter and not a functional dependence for characterization of the changes in the coda wave.

In many cases, it is useful to characterize the difference between two codas by a single number, for instance, by a slope $\alpha_{0}$ of the first quasi-monotonous front of $\tau_{0}\left(t_{c}\right), t_{1}<t_{c}<t_{2}$. It is possible to show [2,31] that parameter $\alpha_{0}$ corresponds to a stretching coefficient $\alpha$ maximizing the correlation coefficient in which shift is replaced by stretching of the time axis, 


$$
S(\alpha)=\frac{\int_{t_{1}}^{t_{2}} r_{i}(t(1+\alpha)) r_{p}(t) d t}{\sqrt{\int_{t_{1}}^{t_{2}} r_{i}^{2}(t(1+\alpha)) d t \cdot \int_{t_{1}}^{t_{2}} r_{p}^{2}(t) d t}} .
$$

The optimal stretching parameter $\alpha$,

$$
\alpha_{0}=\underset{\alpha}{\operatorname{argmax}}(S(\alpha))
$$

and the corresponding decorrelation coefficient

$$
D_{0}=1-\max _{\alpha}(S(\alpha))
$$

do not depend on a window coordinate and can be used as appropriate single-valued criterions of a mismatch between two coda waves.

It should be noted that the non-monotonous behavior of the optimal delay $\tau_{T}\left(t_{c}\right)$ on the window coordinate has been also observed in additional experiments with heating and cooling of the sample while the average slope of the curve tended to the positive (for the heating) or to the negative (for the cooling) value. Similar coda wave types are reported in [2] and in [10].

For the delayed pump and probe, their interaction is due to the aftereffect quantified here by parameters $D_{0}$ and $\alpha_{0}$. It is of interest to estimate the aftereffect magnitude by varying the time delay ( $T_{\text {delay, }}$ see Fig. 5) between the pump and probe waves. As one can observe, when $T_{\text {probe }}>\tau_{\text {pump }}$ the decay of the curves is not instantaneous but exhibits a relaxation behavior. In particular, for $T_{\text {delay }}=1 \mathrm{~ms}$ that exceeds $\tau_{\text {pump }}=0.8 \mathrm{~ms}$ by $0.2 \mathrm{~ms}$, the stretching parameters decrease by 6.5 and $15 \mathrm{~dB}$ for $\alpha_{0}$ and $D_{0}$ respectively. In the opposite case where the pump and probe signals overlaps ( $T_{\text {delay }}<0.8 \mathrm{~ms}$ ), the values of $\alpha_{0}$ and $D_{0}$ are considerably perturbed by the random residual HF pump component that still remains after the application of the subtraction procedure. A greater decay rate of $D_{0}$ in comparison to $\alpha_{0}$ can be explained by quadratic dependence of $D_{0}$ on pump amplitude while the dependence of $\alpha_{0}$ is linear [10]. This is typical for the case of quadratic hysteretic nonlinearity associated to the presence of cracks.
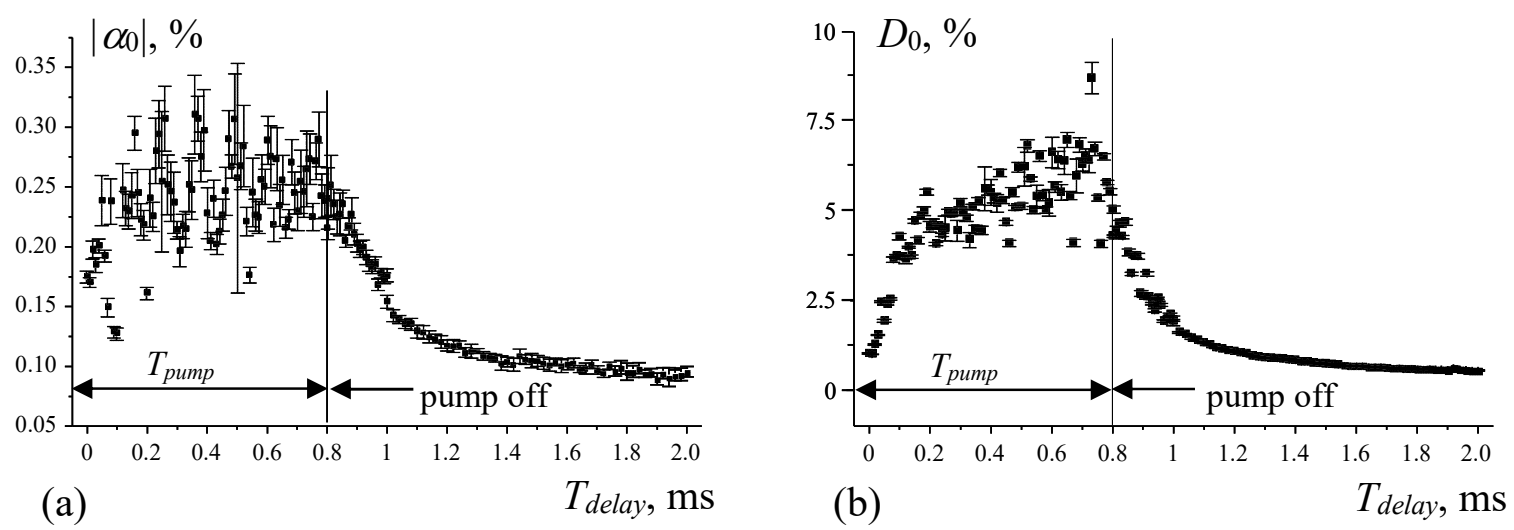
Fig. 7. Stretching parameters $\alpha_{0}$ (a) and $D_{0}$ (b) as functions of probe delay $T_{\text {delay. }}$. The vertical line indicates the moment when the pump is switched off.

A most plausible mechanism for the aftereffect is the finite decay rate of the pump wave. This means that even after switching off the LF pump signal, the remaining LF reverberations play the role of the pump and induce frequency mixing. Although we do not measure the pump wave reverberations directly in a similar way as it is done for the probe signal in our experiments, the both reverberation times are linked through the quality factor of the material. For a broad class of material with many inner contacts [32], the quality factor is approximately constant which means that the frequency factor between the high and low frequencies should be equal to the factor between the corresponding reverberation times. The pump frequency $76.88 \mathrm{kHz}$ and the probe wave signal being a linear frequency sweep in the range $0.5-1 \mathrm{MHz}$ with the center at $750 \mathrm{kHz}$ produce a factor of 9.8. The same value with a good precision can be retrieved from Fig 6(a) (probe signal decay time of $100 \mu \mathrm{s})$ and from Fig. 7 (a,b) in which the aftereffect duration is estimated as $1 \mathrm{~ms}$.

In conclusion, the results in Fig. 7 demonstrate the applicability of the method even in the case when the pump wave can not be fully filtered out of the measured coda due to technical or other reasons. At the same time, the entirely damaged sample with a high crack number and density represents an easy case for the CWI technique. In the next section, experiments on a locally damaged sample are described.

\section{Investigative CWI regime on the locally damaged sample}

\subsection{Pump and probe signals in the continuous mode}

In a locally damaged sample the deterioration degree is much less that the one of the entirely damaged sample. In addition, the sample geometry is much more ordered that results in more ordered and less chaotic resonance patterns. In this case, it is preferable to use a pump sweep instead of a harmonic LH excitation in order to make a more complex resonance structure that corresponds to multiple reverberations of a diffused type. The LF excitation by a chain of sweeps $(50-250 \mathrm{kHz}$ frequency range and $2 \mathrm{~ms}$ duration) make it possible to use a continuous asynchronous scheme (Fig. 8) in which the probe sweeps are excited each time at a different moment relative to the pump sweep beginning. In this situation, the parasite HF generation is removed by averaging. To increase overall pump power, we set $T_{\text {pause }}=0$ and launch pump sweeps 
with no pauses, since averaging efficiently replaces the aftereffect which become masked here by the subsequent pump sweep generations.

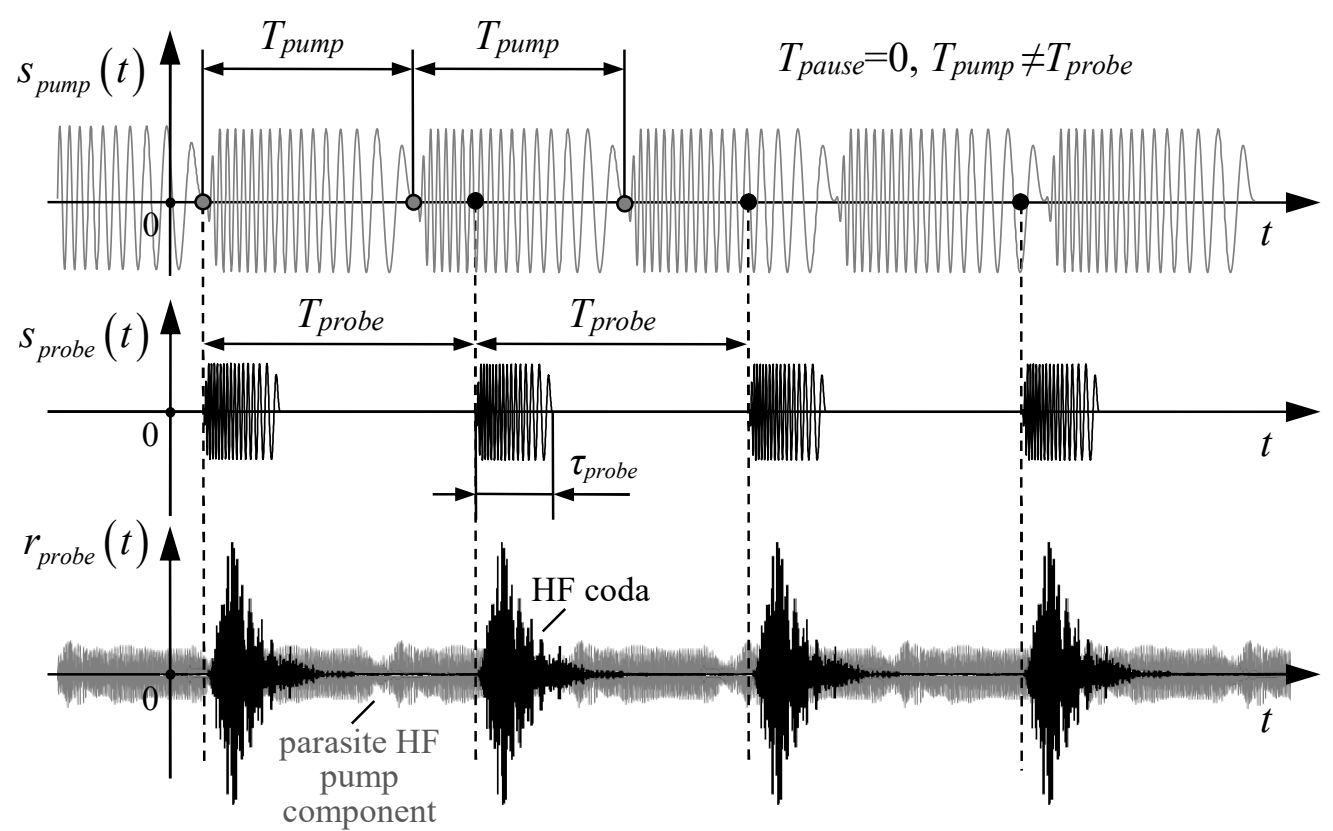

Fig. 8. Illustration for emitted pump and probe signals $s_{\text {pump }}(t)$ and $s_{\text {probe }}(t)$, and for the received coda wave $r_{\text {probe }}(t)$ in the presence of the continuous pump, in the experiment on the locally damaged sample in the investigative regime.

\subsection{Correlation analysis}

Windowed correlation analysis results are plotted in Fig. 9. Despite that the overall degree of deterioration is considerably weaker for the locally damaged sample than for the entirely damaged one, both decorrelation coefficient and optimal delay are still considerably affected by the nonlinear interaction between pump and probe. However, the behavior of curves is totally different. In the latter case, the decorrelation coefficient $D_{T}\left(t_{c}\right)$ gradually decreases as a function of the window position $t_{c}$; the decay reflects the fact that the information on local properties change due to pumping is accumulated with the growing number of reverberations and scattering acts. The corresponding optimal delay $\tau_{T}\left(t_{c}\right)$ is linear on $t_{c}$ which means that the windowed pieces of the perturbed coda arrive with a linearly increasing delay relative to its copies with no pumping (and also change their shape since $D_{T}\left(t_{c}\right)$ decays).

The linear delay effect is possible to understand by analogy with the case of ordinary CWI in which pumping is absent and the coda waves are measured on the intact and damaged samples. 
The presence of damage in one small region effectively lowers the elasticity moduli in that region thus locally decreasing the sound velocity as a material parameter. It can be shown that such a softer zone sensed many times by multiple rays forming coda results in a retardation of various coda fragments, and that the delay almost linearly depends on the arrival time (or on a narrow window central coordinate $t_{c}$ ) [33]. Further, if now the reference and perturbed coda waves are obtained not on the intact and damage samples, but on a damaged sample with and without pumping, as it is in our experiments, the linear delay effect can be attributed to nonlinear softening of the material. Indeed, it is known (see e.g. Fig. 5(b) in [34]) that the sound velocity drop can be or order of $10 \%$ in strongly pumped microdamaged materials, while the CWI technique sensitivity is generally much finer than that value. The linear delay is also obtained by CWI modeling with and without pumping on a sample having a nonlinear inclusion [35].

Thus the correlation analysis (Fig. 9) generally confirms the applicability of the nonlinear CWI in the continuous mode for a sample with local impact damage. To further investigate the question of the technique validity in various conditions, in the next section we describe measurements in the pulsed mode with the aftereffect, for different pump amplitudes, and for the intact and locally damaged sample. 


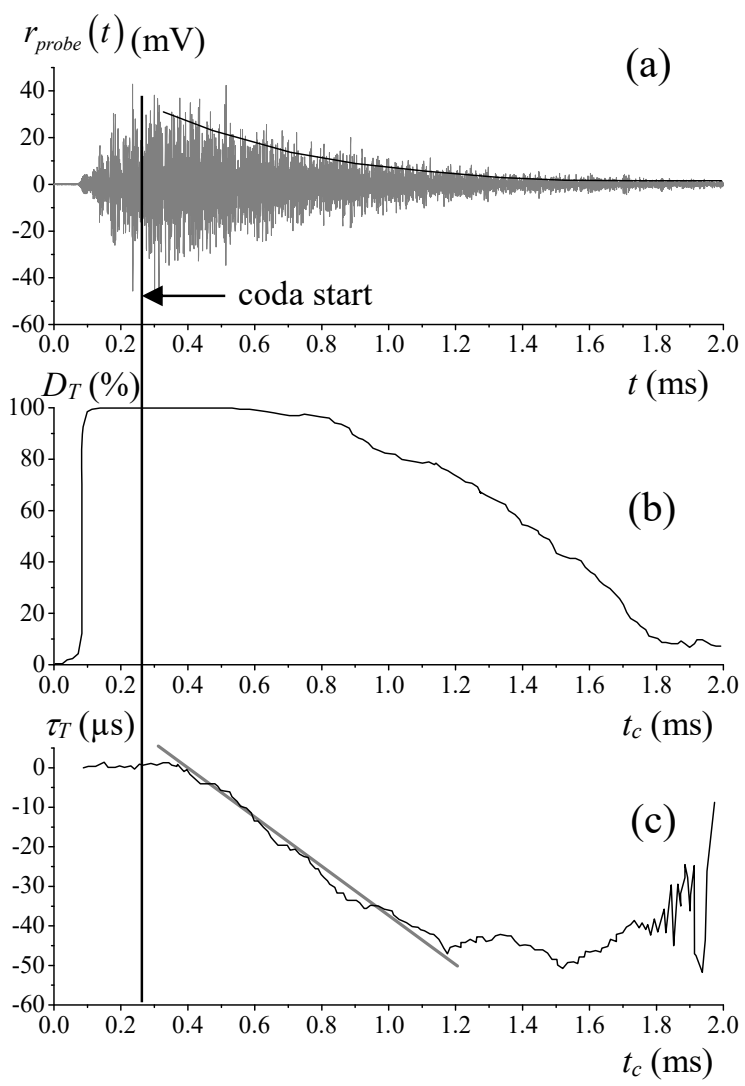

Fig. 9. Reverberation signal (a) obtained on the locally damaged glass plate (continuous mode) and the criterions $D_{T}\left(t_{c}\right)$ and $\tau_{T}\left(t_{c}\right)((\mathrm{b})$ and (c), respectively) characterizing the deviation between two coda waves measured with and without pumping. The inclined line in plot (c) has the slope $\alpha_{0}$ (see Eq. (6)). The window width $T=150 \mu$ s.

\subsection{Nonlinear CWI applicability in pulsed and continuous modes}

This section is concerned with a bunch of nonlinear CWI experiments in the investigative regime in the pulsed and continuous modes. More precisely, the influence of the pump amplitude on the standard CWI criterions in these modes are studied and it is shown that even for moderate pump intensities the criterions clearly reveal the presence of damage. To do so, all measurements was performed on the locally damaged sample and on the intact sample of the same material and dimensions. The pumping protocol used comprised 20 equal steps increasing pump level from 0 to $160 \mathrm{~V}$. After the pump has been maintained at the maximum level for the five subsequent steps. Five final measurement steps were carried out with the pump being turn off. These steady state measurements were set up in order to estimate possible slow dynamics effects. The protocol has 
been adopted from [10]. Each measurement step consisted in 10 averaged coda acquisitions and lasted about $2 \mathrm{~s}$. Between each measurement step the pump was switched off for $0.5 \mathrm{~s}$.

For each excitation protocol in terms of pump and probe, all time part meters were selected exactly as in the previous experiments (see Figs. 5, 8 and parameters definitions in the appropriate Sections). Since here pump amplitude dependencies in various cases are studied, single-valued criterions $D_{0}$ and $\alpha_{0}$ introduced in Eqs. (6) and (7) are used. Experimental results shown in Fig. 10 indicate that $\alpha_{0}$ is four times higher $(0.025 \%$ vs $0.1 \%)$ and $D_{0}$ is approximately two times higher $(0.07 \%$ vs $0.12 \%)$ in continuous mode than in the pulsed one, due to the obvious difference on the overall injected pump energy in the two modes.

After switching off the pump, $\alpha_{0}$ and $D_{0}$ demonstrate different behaviors in these modes. In the pulsed mode, the criterions immediately return to zero, while in the continuous mode they quickly drop to a nonzero value and then slowly decrease. A plausible explanation is in thermal nature of the effect when on the pulsed mode the sample cools down during the pauses whereas in the continuous mode the temperature generally increases the effect.

No pump-induced changes were observed in the intact sample within the experimental precision. Thus the results of this Section indicate that the nonlinear CWI is capable of defect detection in a number of situations with various pump-probe schemes.
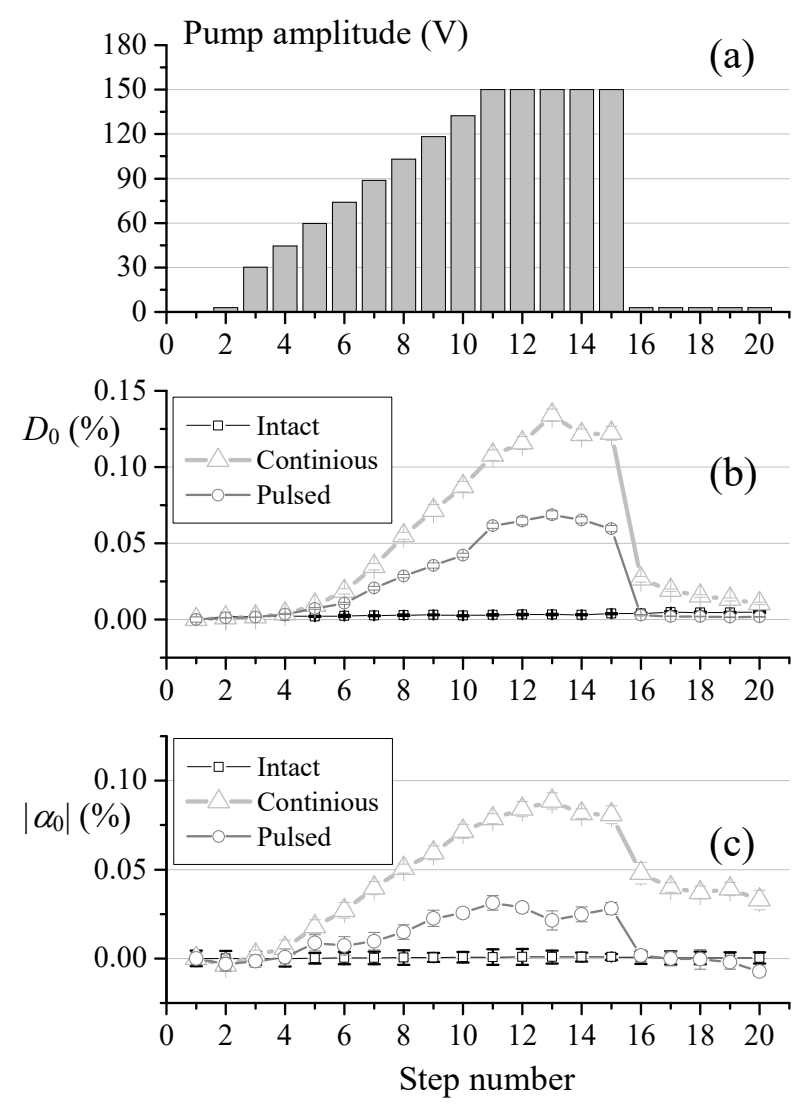
Fig. 10. (a) Pumping protocol used for nonlinear CWI in the extensive series of experiments in the investigative regime. Decorrelation coefficient $D_{0}$ and optimal stretching parameter $\alpha_{0}($ (b) and (c), respectively) in the continuous (triangles) and pulsed modes (circles); comparison is given for an intact glass sample (squares).

\section{Localizing a defect with the TR pumping}

In order to localize the defect in the glass plate locally damaged by impact with a local crack (Fig. 4), the nonlinear CWI technique was combined with the TR process as described in Section 2.2. The retro-focused pump was provided by the following procedure:

$\circ$ broadcasting of the primary pump wave $s_{\text {pump }}(t)$ in the pulsed mode and with the amplitude of $150 \mathrm{~V}$ i.e. as indicated in Fig. 5 with the parameters from Section $5.1(50-250 \mathrm{kHz}$ frequency sweep range and $2 \mathrm{~ms}$ duration);

$\circ$ recording pump reverberations $r_{1 \text { pump }}(t)$ at point $r_{1}$;

$\circ$ re-broadcasting of the secondary pump wave $q_{1 \text { pump }}(t)$ calculated via Eq. (1) with $r_{\text {pump }}(t)$ substituted by $r_{1 \text { pump }}(t)$ in there with the period $T_{\text {pump }}=20 \mathrm{~ms}$. In order to increase the nonlinear interaction, the time-reverse LF pump is rebroadcasted in continuous mode.

$\circ$ the probe coda $(500-1000 \mathrm{kHz}$ frequency sweep range and $100 \mu \mathrm{s}$ duration) is emitted with the period $T_{\text {probe }}=17 \mathrm{~ms} \neq T_{\text {pump }}$.

The result of the procedure is shown in Fig. 11 where $q_{1 \text { pump }}(t)$ is displayed together with continuous pump waveforms: $r_{1 \text { pump } 1}(t)$ measured at point $r_{1}$ i.e. at focus and $r_{2 \text { pump } 1}(t)$ measured at point $r_{2}$ i.e. out of focus. One can see that focusing actually means that at some periodically repeating moments equal peaks appear in $r_{1 \text { pump } 1}(t)$ record while at the out-of-focus positions such peaks are absent despite some acoustic energy is still present. 


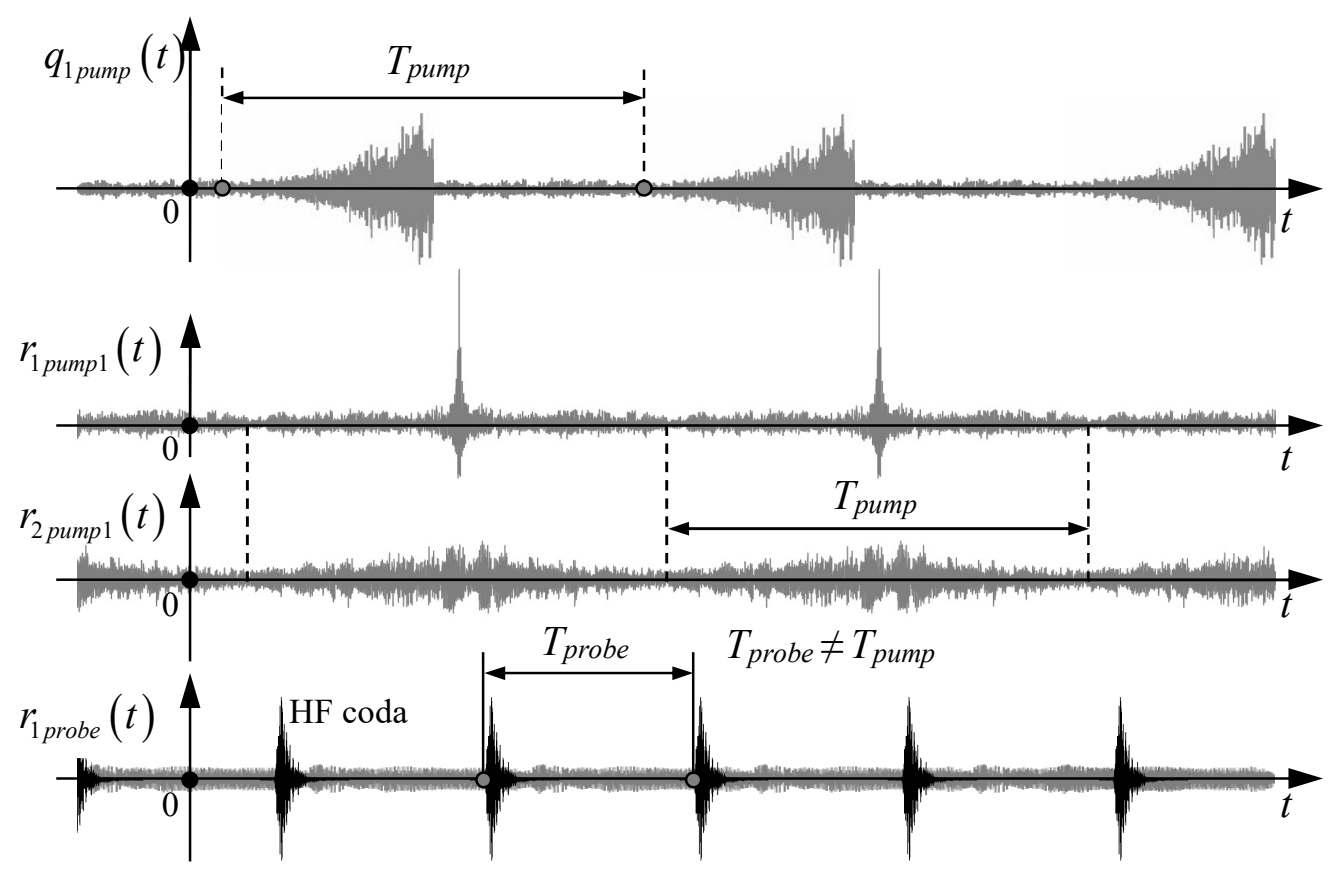

Fig. 11. Re-broadcasted pump signal $q_{1 \text { pump }}(t)$ inversely filtered accordingly to Eq. (1) for retrofocusing at location $r_{1}$ of the defect (see Fig. 4), pump signal $r_{1 \text { pump } 1}(t)$ recorded at the focal point $r_{1}$, pump signal $r_{1 \text { pump } 2}(t)$ received at $r_{2}$ out of focal point, and asynchronous $\left(T_{\text {probe }} \neq T_{\text {pump }}\right)$ high frequency probe signal $r_{1 \text { probe }}(t)$ measured at $r_{1}$.

In order to demonstrate the localization capability of the TR-based nonlinear CWI, it is necessary to focus pump not only on the defect, but also on some other positions in the sample where material is intact. To do so, we repeated the above measurements for other positions $r_{2}$ and $r_{3}$ in order to have a matrix $r_{i \text { pump } j}(t)$ of pump signals with $i, j=1 . .3$. Moreover, to have more reliable data, we shifted several times the positions of received $r_{2}$ and $r_{3}$ around the focal point $r_{1}$ in a random manner. Then for each set of measurements the stretching criterions Eqs. (6), (7) with the values of parameters based on the analysis given in Section 5.2 (Fig. 9): the starting position of the CWI window is $250 \mu \mathrm{s}$ and the duration is $1 \mathrm{~ms}$. For all out-of-defect positions the results were averaged. Measurements show that TR process allowed obtaining RMS signal contrast of $3.8 \mathrm{~dB}$ and amplitude contrast of $11.4 \mathrm{~dB}$. 


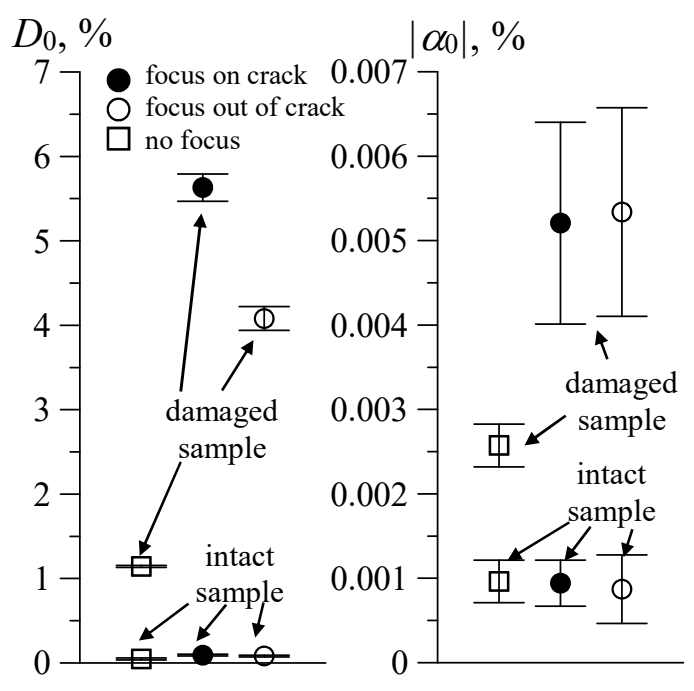

Fig. 12. Evolution of the stretching parameter $\alpha_{0}$ and of decorrelation coefficient $D_{0}$ measured on the locally damaged sample when the pump wave is not focused, focused at the crack and outside of it. Reference measurements correspond to the intact sample of the same geometry.

The calculated stretching criterions are illustrated in Fig. 12. For the intact sample, no decorrelation was seen $\left(D_{0} \approx 0\right)$ while for the locally damaged sample it is certainly present. With no TR procedure applied i.e. in the investigative regime, a $1 \%$ decorrelation is obtained indicating the presence of damage. Further, the decorrelation increases when retro-focusing is done, and the situation when the focus coincides with the defect position is clearly differs from the case of focusing at intact material regions ( $D_{0} \approx 6 \%$ and $D_{0} \approx 4 \%$, respectively). Although affected by an imperfect retro-focusing procedure that uses only one transducer for the calculation of the appropriate secondary pumping waveform, this result demonstrates the localization potential of the method.

\section{Summary and conclusions}

This paper contributes to the development of nonlinear CWI method of nondestructive testing in which pump and probe waves interact due to the presence of contact acoustic nonlinearity which, in turn, indicates damage in materials. Due to nonlinear wave interactions, HF coda wave created by multiple reverberations of the probe pulse is affected by LF pumping. The slight differences in the coda measured in the absence and presence of pumping are accumulated in the course of reverberation process and are quantified using the local (windowed) correlation function together with the corresponding optimal delay. A fist monotonous front of the delay as a function of 
the window center coordinate produces another criterion of damage occurrence in a global rather than local form i.e. as a single parameter and not a function.

As it was already suggested, the method can be used for the detection of a global damage presence which is called investigative regime in this paper. A new step forward made here is the addition of the localization option which uses the time reversal principle. The localization regime does not require any hardware modification and consists in the generation of a secondary pump wave that focuses at a given location in the material. By moving the focal spot, it is possible to selectively interrogate various locations and finally to determine the damage position.

Our measurements indicate that nonlinear response of a damaged zone is significantly higher than the one of neighboring intact regions in the material even in the case of one-channel retro-focusing. This is indeed a strict minimum in terms of experimental setup, since, theoretically, good focusing is guaranteed only when an entire wavefront is inverted. Thus, improvement of TR quality by adding additional channels could increase the performances of this NDT method. Multichannel pumping can be done in asynchronous mode in order to maintain more uniform energy contrast in time domain. Some improvement can also be achieved by optimizing the chaotic cavity.

At the same time, with all the encountered technical difficulties and limitations, the reported results demonstrate feasibility of the method and suggest an appropriate configuration for health monitoring of complex structures.

\section{Acknowledgements}

The research leading to these results has gratefully received funding from the European Union Seventh Framework Program (FP7/2007-2013) under Grant Agreement n 314768 (ALAMSA). One of us (Vladislav Aleshin) particularly acknowledges the support from the Tomsk State University competitiveness improvement program.

\section{References}

[1] K. Aki, B. Chouet, Origin of coda waves: Source, attenuation, and scattering effects, J. Geophys. Res. 80 (1975) 3322-3342. https://doi.org/10.1029/JB080i023p03322.

[2] R. Snieder, A. Grêt, H. Douma, J. Scales, Coda Wave Interferometry for Estimating Nonlinear Behavior in Seismic Velocity, Science. 295 (2002) 2253-2255. https://doi.org/10.1126/science.1070015.

[3] P. Fröjd, P. Ulriksen, Frequency selection for coda wave interferometry in concrete structures, Ultrasonics. 80 (2017) 1-8. https://doi.org/10.1016/j.ultras.2017.04.012.

[4] T. Planès, E. Larose, A review of ultrasonic Coda Wave Interferometry in concrete, Cem. Concr. Res. 53 (2013) 248-255. https://doi.org/10.1016/j.cemconres.2013.07.009. 
[5] Y. Zhang, T. Planès, E. Larose, A. Obermann, C. Rospars, G. Moreau, Diffuse ultrasound monitoring of stress and damage development on a 15-ton concrete beam, J. Acoust. Soc. Am. 139 (2016) 1691-1701. https://doi.org/10.1121/1.4945097.

[6] B. Chen, D. Callens, P. Campistron, E. Moulin, P. Debreyne, G. Delaplace, Monitoring cleaning cycles of fouled ducts using ultrasonic Coda Wave Interferometry (CWI), Ultrasonics. (2018). https://doi.org/10.1016/j.ultras.2018.12.011.

[7] E. Larose, S. Hall, Monitoring stress related velocity variation in concrete with a $2 \times 10-5$ relative resolution using diffuse ultrasound, J. Acoust. Soc. Am. 125 (2009) 1853-1856. https://doi.org/10.1121/1.3079771.

[8] E. Niederleithinger, C. Wunderlich, Influence of small temperature variations on the ultrasonic velocity in concrete, in: Denver, Colorado, USA, 2013: pp. 390-397. https://doi.org/10.1063/1.4789074.

[9] Y. Zhang, O. Abraham, V. Tournat, A. Le Duff, B. Lascoup, A. Loukili, F. Grondin, O. Durand, Validation of a thermal bias control technique for Coda Wave Interferometry (CWI), Ultrasonics. 53 (2013) 658-664. https://doi.org/10.1016/j.ultras.2012.08.003.

[10] Y. Zhang, V. Tournat, O. Abraham, O. Durand, S. Letourneur, A. Le Duff, B. Lascoup, Nonlinear mixing of ultrasonic coda waves with lower frequency-swept pump waves for a global detection of defects in multiple scattering media, J. Appl. Phys. 113 (2013) 064905. https://doi.org/10.1063/1.4791585.

[11] E. Larose, A. Obermann, A. Digulescu, T. Planès, J.-F. Chaix, F. Mazerolle, G. Moreau, Locating and characterizing a crack in concrete with diffuse ultrasound: A four-point bending test, J. Acoust. Soc. Am. 138 (2015) 232-241. https://doi.org/10.1121/1.4922330.

[12] T. Planès, E. Larose, V. Rossetto, L. Margerin, Imaging multiple local changes in heterogeneous media with diffuse waves, J. Acoust. Soc. Am. 137 (2015) 660-667. https://doi.org/10.1121/1.4906824.

[13] S. Haupert, G. Renaud, A. Schumm, Ultrasonic imaging of nonlinear scatterers buried in a medium, NDT E Int. 87 (2017) 1-6. https://doi.org/10.1016/j.ndteint.2016.12.010.

[14] P.-Y. Le Bas, M.C. Remillieux, L. Pieczonka, J.A. Ten Cate, B.E. Anderson, T.J. Ulrich, Damage imaging in a laminated composite plate using an air-coupled time reversal mirror, Appl. Phys. Lett. 107 (2015) 184102. https://doi.org/10.1063/1.4935210.

[15] M. Fink, D. Cassereau, A. Derode, C. Prada, P. Roux, M. Tanter, J.-L. Thomas, F. Wu, Timereversed acoustics, Rep. Prog. Phys. 63 (2000) 1933-1995. https://doi.org/10.1088/00344885/63/12/202.

[16] C. Payan, T.J. Ulrich, P.-Y. Le Bas, T. Saleh, M. Guimaraes, Probing materials damage at various depths by use of Time Reversal Elastic Nonlinearity Diagnostic: Application to concrete., Proc. Meet. Acoust. 16 (2012) 045013. https://doi.org/10.1121/1.4764477.

[17] T.J. Ulrich, A.M. Sutin, T. Claytor, P. Papin, P.-Y. Le Bas, J.A. TenCate, The time reversed elastic nonlinearity diagnostic applied to evaluation of diffusion bonds, Appl. Phys. Lett. 93 (2008) 151914. https://doi.org/10.1063/1.2998408.

[18] T.J. Ulrich, P.A. Johnson, R.A. Guyer, Interaction Dynamics of Elastic Waves with a Complex Nonlinear Scatterer through the Use of a Time Reversal Mirror, Phys. Rev. Lett. 98 (2007) 104301. https://doi.org/10.1103/PhysRevLett.98.104301.

[19] K. Van Den Abeele, Multi-mode nonlinear resonance ultrasound spectroscopy for defect imaging: An analytical approach for the one-dimensional case, J. Acoust. Soc. Am. 122 (2007) 73-90. https://doi.org/10.1121/1.2735807.

[20] P. Fröjd, P. Ulriksen, Amplitude and phase measurements of continuous diffuse fields for structural health monitoring of concrete structures, NDT E Int. 77 (2016) 35-41. https://doi.org/10.1016/j.ndteint.2015.10.003.

[21] Y. Zhang, V. Tournat, O. Abraham, O. Durand, S. Letourneur, A. Le Duff, B. Lascoup, Nonlinear coda wave interferometry for the global evaluation of damage levels in complex solids, Ultrasonics. 73 (2017) 245-252. https://doi.org/10.1016/j.ultras.2016.09.015. 
[22] M. Tanter, J.-L. Thomas, M. Fink, Time reversal and the inverse filter, J. Acoust. Soc. Am. 108 (2000) 223-234. https://doi.org/10.1121/1.429459.

[23] B.E. Anderson, J. Douma, T.J. Ulrich, R. Snieder, Improving spatio-temporal focusing and source reconstruction through deconvolution, Wave Motion. 52 (2015) 151-159. https://doi.org/10.1016/j.wavemoti.2014.10.001.

[24] E. Herth, F. Lardet-Vieudrin, F. Deux, L. Valbin, E. Algré, J. Schell, H. Steger, Detection of out-of-plane and in-plane (XYZ) motions of piezoelectric microcantilever by 3D-Laser Doppler Vibrometry, in: 2016 Symp. Des. Test Integr. Packag. MEMSMOEMS DTIP, 2016: pp. 1-4. https://doi.org/10.1109/DTIP.2016.7514876.

[25] C. Draeger, M. Fink, One-Channel Time Reversal of Elastic Waves in a Chaotic 2D-Silicon Cavity, Phys. Rev. Lett. 79 (1997) 407-410. https://doi.org/10.1103/PhysRevLett.79.407.

[26] C. Draeger, M. Fink, One-channel time-reversal in chaotic cavities: Theoretical limits, J. Acoust. Soc. Am. 105 (1999) 611-617. https://doi.org/10.1121/1.426251.

[27] C. Draeger, J.-C. Aime, M. Fink, One-channel time-reversal in chaotic cavities: Experimental results, J. Acoust. Soc. Am. 105 (1999) 618-625. https://doi.org/10.1121/1.426252.

[28] R.L. Weaver, W. Sachse, Diffusion of ultrasound in a glass bead slurry, J. Acoust. Soc. Am. 97 (1995) 2094-2102. https://doi.org/10.1121/1.412002.

[29] J.E. Michaels, T.E. Michaels, Detection of structural damage from the local temporal coherence of diffuse ultrasonic signals, IEEE Trans. Ultrason. Ferroelectr. Freq. Control. 52 (2005) 1769-1782. https://doi.org/10.1109/TUFFC.2005.1561631.

[30] J.F. Peters, M. Muthuswamy, J. Wibowo, A. Tordesillas, Characterization of force chains in $\begin{array}{lllllll}\text { granular material, } & \text { Phys. } & \text { Rev. } & \text { E. } & 72 & \text { (2005) }\end{array}$ https://doi.org/10.1103/PhysRevE.72.041307.

[31] O.I. Lobkis, R.L. Weaver, Coda-Wave Interferometry in Finite Solids: Recovery of P-to-S Conversion Rates in an Elastodynamic Billiard, Phys. Rev. Lett. 90 (2003) 254302. https://doi.org/10.1103/PhysRevLett.90.254302.

[32] A.C. Kibblewhite, Attenuation of sound in marine sediments: A review with emphasis on new low-frequency data, J. Acoust. Soc. Am. 86 (1989) 716-738. https://doi.org/10.1121/1.398195.

[33] C. Pacheco, R. Snieder, Time-lapse travel time change of multiply scattered acoustic waves, J. Acoust. Soc. Am. 118 (2005) 1300-1310. https://doi.org/10.1121/1.2000827.

[34] L.A. Ostrovsky, P.A. Johnson, Dynamic nonlinear elasticity in geomaterials, Nuovo Cimento Riv. Ser. 24 (2001) 1-46.

[35] G. Chen, D. Pageot, J.-B. Legland, O. Abraham, M. Chekroun, V. Tournat, Numerical modeling of ultrasonic coda wave interferometry in a multiple scattering medium with a localized nonlinear defect, Wave Motion. 72 (2017) 228-243. https://doi.org/10.1016/j.wavemoti.2017.03.004. 